\title{
Genotype by Environment Interaction: The Greatest Obstacle in Precise Determination of Rice Sheath Blight Resistance in the Field
}

Yuxiang Zeng, State Key Laboratory of Rice Biology, China National Rice Research Institute, Hangzhou, 310006, P. R. China; Junsheng Shi, Seed Management Station of Zhengjiang Province, Hangzhou, 310020, P. R. China; and Zhijuan Ji, Zhihua Wen, Yan Liang, and Changdeng Yang, ${ }^{\dagger}$ State Key Laboratory of Rice Biology, China National Rice Research Institute, Hangzhou, 310006, P. R. China

\begin{abstract}
Rice sheath blight (SB) is the most serious rice disease in China. Resistance of rice to SB is a quantitative trait that is easily influenced by the environment; however, the extent of environmental influence on SB field resistance is still poorly understood. To identify rice genotype by environment interactions for SB resistance, 211 rice genotypes originating from 15 countries were planted and evaluated for SB field resistance in six different environments between 2012 and 2016 after inoculation with the SB pathogen isolate ZJ03. In addition, 65 rice genotypes were evaluated for SB field resistance in another four environments between 2013 and 2016 using ZJ03.

Variations in SB field resistance were observed in different genotypes in different environments using objective and subjective rating methods. Two-way analysis of variance indicated that the interaction between the genotype and environment had a highly significant effect on SB field resistance. This analysis indicated that the environment had more of an influence than the genotype itself on SB field resistance, and the genotype by environment interaction was the greatest obstacle in obtaining a precise determination of SB field resistance in rice. The most resistant genotype, GD66, is a good candidate for genetic studies and breeding.
\end{abstract}

Sheath blight (SB), caused by Rhizoctonia solani Kühn, is generally considered one of the three major rice diseases second only to rice blast (Park et al. 2008). However, in the past decade, the losses caused by SB have exceeded those caused by rice blast or bacterial blight in China, making it the most serious disease in the largest rice producing country worldwide (Zeng et al. 2011; Zuo et al. 2008, 2014 b). SB reduces rice yields by 8 to $50 \%$ in the rice growing countries of Asia and it causes approximately $20 \%$ loss of rice yields in India (Ghosh et al. 2016). SB is also considered the most common rice disease in the southern United States (Groth and Bond 2007).

No rice species (Oryza sativa L.) or its wild relatives have been found to be immune to the SB pathogen, although some cultivars have varying levels of natural resistance (Srinivasachary et al. 2011). The application of fungicide is a common practice to control SB (Ghosh et al. 2016); however, the application of SB-resistant cultivars is generally considered a more economical and environmentally friendly way to control the disease (Park et al. 2008; Zuo et al. 2014b). Rice SB resistance is a trait controlled by quantitative trait loci (QTLs), and despite extensive studies in the past two decades to find new SB-resistant germplasm and identify SB-resistant QTLs, progress has been very slow and limited compared with rice blast or bacterial blight. A major reason SB is difficult to study is that a number of environmental factors such as sunlight, humidity, temperature, soil fertility, and silicon levels in the soil may contribute to the variation in phenotypic disease resistance (Park et al. 2008).

Recently, QTL analyses have identified a large number of SB resistance QTLs on all 12 rice chromosomes (Srinivasachary et al. 2011; Zeng et al. 2015a), but no more than 10 SB-resistant QTLs were confirmed with observable contributions to SB resistance (Xue et al. 2016). None of the SB resistance QTLs have been cloned by map-based cloning strategies except QTL qSBR11-1, which was mapped to a $0.85-\mathrm{Mb}$ region on chromosome 11 by primary mapping

${ }^{\dagger}$ Corresponding author. E-mail:yangchangdeng@126.com

Y. X. Zeng and J. S. Shi contributed equally to this work.

*The $\boldsymbol{e}$-Xtra logo stands for "electronic extra" and indicates that three supplementary figures and 10 supplementary tables are published online.

Accepted for publication 19 May 2017.

C 2017 The American Phytopathological Society
(Channamallikarjuna et al. 2010) and then cloned based on the information from 11 tandem repeats of the chitinase genes located within this region (Richa et al. 2016).

Genotype by environment interaction is a phenomenon that genotype effects may significantly differ across environments (Zhao and $\mathrm{Xu}$ 2012). Significant genotype by environment interactions have been reported in many plant diseases including black point disease in wheat (Kilic et al. 2009), anthracnose in water yam (Egesi et al. 2009), gray leaf spot disease in maize (Sibiya et al. 2012), net blotch disease in barley (Pinnschmidt and Hovmøller 2002), rice blast (Bonman 1992; Talukder et al. 2005), and rice bacterial blight (Lussewa et al. 2016).

It is well known that SB field resistance is easily affected by the environment; however, it is still unclear how much the genotype by environment interaction influences the determination of SB field resistance. Does the genotype have more of an effect on the determination of SB field resistance than the environment? The answer to this question is very important because if the genotype by environment interaction is significant, it directly affects the identification of SB resistance QTLs. To examine the effect of the genotype by environment interaction on SB field resistance, we examined the SB field resistance of 211 rice genotypes originating from more than 15 countries grown in six different environments from 2012 to 2016. We also examined SB field resistance of an additional 65 rice genotypes in four different environments between 2013 and 2016 in order to mine SB resistant restorer or maintainer lines for breeding. SB field resistance data of 65 additional genotypes were also used in the analysis of genotype by environment interaction.

\section{Materials and Methods}

Two hundred and eleven rice genotypes were evaluated for SB field resistance for six environments. The 211 rice genotypes used in this study originated from or were developed in 15 countries including China, the U.S., France, Korea, Japan, Philippines, India, Italy, Indonesia, Thailand, Vietnam, Malaysia, Bangladesh, Nigeria, and Sri Lanka, and are summarized in Supplementary Table S1. The 211 rice genotypes consisted of landraces, cultivars, breeding lines, deep-water rice, upland rice, weedy rice, O. sativa/O. rufipogon descendants, japonica, indica, and javanica, and represent the diversity of rice genetic variation.

Lesion mimic mutant 3 (Lm3) and Lowseedset1 were provided by Prof. Chunhai Shi at Zhejiang University. The eight restorer lines, GD14, GD54, GD61, GD51, GD52, GD66, GD71, and GD120, were 
developed by Changdeng Yang at China National Rice Research Institute (CNRRI). The other 201 genotypes were provided by Xinghua Wei from the collection of the National Midterm Rice Genebank in CNRRI.

The 211 rice genotypes were planted across six different environments for the evaluation of SB resistance. Five of the environments were represented by different years on the same farm and the sixth in a different location for one year. They were planted over five consecutive years: (1) May 2012 in the CNRRI farm in Fuyang, Hangzhou $\left(30^{\circ} 07^{\prime} \mathrm{N}, 119^{\circ} 95^{\prime} \mathrm{E}\right)$, (2) November 2012 in the CNRRI trial station in Lingshui, Hainan $\left(18^{\circ} 48^{\prime} \mathrm{N}, 110^{\circ} 02^{\prime} \mathrm{E}\right)$, (3) May 2013 at the CNRRI farm in Fuyang, Hangzhou, (4) May 2014 in the CNRRI farm in Fuyang, Hangzhou, (5) May 2015 in the CNRRI farm in Fuyang, Hangzhou, and (6) May 2016 in the CNRRI farm in Fuyang, Hangzhou. Field management included all the common agronomic practices of Hangzhou and Hainan regions except that pesticides and fungicides were not used 10 days before inoculation or until SB resistance data were completed in each year.

Sixty-five rice genotypes evaluated for SB field resistance over four years. The 65 rice genotypes used in this study consisted of 17 maintainer lines, 29 restorer lines, 12 landraces, and six cultivars. Among these 65 genotypes, one genotype was from Korea, and 63 genotypes were from more than eight provinces/regions in China. Of those originating from China, 43 genotypes listed in Supplementary Table S2 from Neixiang85B to R-527, were provided by Guanfu Fu of the CNRRI; the other 22 genotypes were provided and preserved by Xinghua Wei at the National Midterm Rice Genebank in CNRRI.

These 65 genotypes were planted over four consecutive years beginning November 2013 at the CNRRI trial station in Lingshui, Hainan $\left(18^{\circ} 48^{\prime} \mathrm{N}, 110^{\circ} 02^{\prime} \mathrm{E}\right)$, and at the CNRRI farm in Fuyang, Hangzhou $\left(30^{\circ} 07^{\prime} \mathrm{N}, 119^{\circ} 95^{\prime} \mathrm{E}\right)$ each May $2014-16$. Field management was the same as mentioned above.

Pathogen inoculation. $R$. solani isolate $\mathrm{ZJ} 03$ was collected in Fuyang, Hangzhou, China, deposited in the State Key Laboratory of Rice Biology, and used for inoculation of rice genotypes in this study. Isolate ZJ03 has been used for mapping SB resistance QTLs in two previous studies (Wen et al. 2015; Zeng et al. 2015b). We followed the inoculation method described by Zou et al. (2000) with minor modification: truncated bamboo-toothpicks 2 to $2.5 \mathrm{~cm}$ long were incubated with ZJ03 on Petri plates containing potato dextrose agar medium in darkness for 7 days at $28^{\circ} \mathrm{C}$. Then, toothpicks covered with mycelium were used to penetrate the third rice leaf sheath, counting from the top at the late tillering stage. At this stage of growth, the second sheath from the top was no longer elongating; therefore, the toothpick remained stable inside the third sheath (Xue et al. 2016). SB resistance was recorded 30 days after inoculation.

Evaluation of SB resistance under field conditions. For each of the inoculation environments, 18 individual plants from each rice genotype were grown in one plot per genotype, arranged with six plants in three rows spaced $17 \mathrm{~cm}$ apart, and with $20 \mathrm{~cm}$ between the rows. The locations of plots were completely randomized. In each plot, three plants in the middle row were inoculated. Two of the tallest panicles in each of the three plants were inoculated at the late-tillering stage for a combined total of six inoculated panicles for each genotype. Thirty days after inoculation, the lesion length of each panicle was recorded. The lesion length was measured in each inoculated panicle. The three inoculated plants from each genotype were also evaluated for SB resistance using the 0 to 9 visual rating system where 0 indicated that the plant was completely immune to the pathogen, and 9 indicated a dead or collapsed plant (Pinson et al. 2005). The visual rating was determined for each plant 30 days after inoculation. Both lesion length and visual rating were used to obtain a more comprehensive evaluation of SB field resistance.

Statistical analysis. The average lesion length was calculated by averaging six inoculated panicles; the maximum lesion length was the longest lesion length of the six inoculated panicles. In addition, we used both the average visual rating from each group of three plants and the maximum visual rating of the three plants to evaluate $\mathrm{SB}$ resistance.

Standard deviation (SD) was calculated with the STDEV function in Microsoft Excel (Microsoft Office Professional Plus 2010, 32-bit) to evaluate the variation of lesion length and visual rating for each genotype in each inoculation environment. Two-way analysis of variance (ANOVA) was calculated using SAS v. 8.01 (SAS Institute, Cary, NC, U.S.A.) to test whether the interaction between the genotype and its environment had a significant effect on SB resistance.

Resistance data for genotypes in some environments were not available for further analysis (Supplementary Tables S3 and S4), because the inoculated plants were eaten by mice or insects or destroyed for other reasons. Those data were omitted and not used in statistical analysis. Although some data were missing, we were able to collect complete resistance data from 169 genotypes in all six environments, as well as complete data from the 56 genotypes that were cultivated in four different environments.

Cluster analysis was conducted using IBM SPSS Statistics software (version 22) with the Hierarchical Cluster Analysis program. The cluster methods used were the 'between-groups linkage' and the Euclidean distance. Six variables were used to group the 211 and the 65 genotypes into clusters, respectively.

\section{Results}

Rice line GD66 was the most resistant genotype against $R$. solani among the 211 rice genotypes evaluated for SB resistance over six environments. As a metric for SB resistance, we calculated the average lesion length, maximum lesion length, average visual rating, and maximum visual rating for each genotype in the six independent plantings. The average values for each genotype were used to determine the rice lines with the most resistant genotypes. When sorted by the average lesion length the five most resistant genotypes were GD66 $(12.6 \mathrm{~cm})$, Yinhesizhan $(13.0 \mathrm{~cm})$, Yuxiangyouzhan $(13.3 \mathrm{~cm})$, Xiangtezhan $(14.3 \mathrm{~cm})$, and Yangdao $4(14.8 \mathrm{~cm})$. The five most resistant genotypes when sorted by average visual rating were GD66 (1.4), Bahagia (1.7), Yinhesizhan (1.8), Zhongshan1 (1.8), and Yangdao 4 (1.8). The five most resistant genotypes sorted by maximum lesion length were GD66 $(18.3 \mathrm{~cm})$, Conglaai $(18.7 \mathrm{~cm})$, Yinhesizhan $(19.7 \mathrm{~cm})$, Yuxiangyouzhan $(20.1 \mathrm{~cm})$, and Yangdao $4(20.3 \mathrm{~cm})$. The five most resistant genotypes sorted by maximum visual rating were GD66 (1.8), Yangdao 4 (2.1), Bahagia (2.1), Zhongshan1 (2.2), and Tetep (2.2). These results show that GD66, an indica restorer line developed by Changdeng Yang at CNRRI, was the most resistant rice genotype to $R$. solani in this study.

The restorer line Chuannong527 had the most SB resistance among the 65 rice genotypes evaluated over four environments. To mine SB resistant maintainer and restorer lines, 65 genotypes, 46 of which were maintainer or restorer lines, were examined for SB resistance over four years. The five most resistant genotypes among the 65 genotypes examined were Chuannong527 $(18.6 \mathrm{~cm})$, Aijiaobaimizai $(20.9 \mathrm{~cm})$, Minghui $63(21.4 \mathrm{~cm})$, Tianhui988 $(21.7 \mathrm{~cm})$, and Hanyangli2 $(23.3 \mathrm{~cm})$ when sorted by average lesion length; Aijiaobaimizai (1.6), Hanyangli2 (1.8), Chuannong527 (1.9), Tianhui988 (2.1), and Zhaitang (2.4) when sorted by average visual rating; Aijiaobaimizai $(24.9 \mathrm{~cm})$, Chuannong527 $(26.5 \mathrm{~cm})$, Minghui63 $(28.4 \mathrm{~cm})$, Hanyangli2 $(30.5 \mathrm{~cm})$, and Xiushui03 $(30.9 \mathrm{~cm})$ when sorted by the maximum lesion length; and Aijiaobaimizai (2), Hanyangli2 (2), Tianhui988 (2.8), Chuannong527 (3.1), and Shuhui527 (3.1) were the most resistant genotypes when sorted by the maximum visual rating over the four years of study. These results indicated that the Chuannong527 was the most resistant restorer line and would be a good candidate for breeding rice for resistance against SB; however, we did not find any maintainer lines with adequate SB resistance in our assessment among the tested genotypes.

Comparison of different methods for evaluating SB field resistance in rice. We used average lesion length, average visual rating, maximum lesion length, and maximum visual rating to evaluate SB resistance of each genotype in our cohorts. The average lesion length or average visual rating represents the average level of resistance for a specific genotype to the pathogen, while the maximum lesion length or visual rating represents the maximum damage a specific genotype suffered after infection. We could not confirm which method was the most adequate for the evaluation of SB resistance, but correlation analysis showed that the four measurements were significantly correlated to each other, with correlation coefficients that were above 0.71 (Tables 1 and 2); suggesting that these four methods were consistent in their evaluation of SB resistance. 
Variation of SB resistance within the same genotype grown in different years. Although SB resistance data for some genotypes were not available for all study environments due to herbivory or plant collapse, we still collected complete data from 169 and 56 genotypes for all study environments (lesion length for 57 genotypes

Table 1. Pearson's correlation coefficients for the four sheath blight evaluation methods used in the assessment of 211 rice genotypes. Data used in these analyses are presented in Supplementary Table S1. ${ }^{\mathrm{a}}$

\begin{tabular}{lcccc}
\hline & $\begin{array}{c}\text { Average } \\
\text { lesion } \\
\text { length }\end{array}$ & $\begin{array}{c}\text { Average } \\
\text { visual } \\
\text { rating }\end{array}$ & $\begin{array}{c}\text { Maximum } \\
\text { lesion } \\
\text { length }\end{array}$ & $\begin{array}{c}\text { Maximum } \\
\text { visual } \\
\text { rating }\end{array}$ \\
\hline Average lesion length & - & - & - & - \\
Average visual rating & $0.77^{* *}$ & - & - & - \\
Maximum lesion length & $0.96^{* *}$ & $0.71^{* *}$ & - & - \\
Maximum visual rating & $0.76^{* *}$ & $0.98^{* *}$ & $0.74^{* *}$ & - \\
\hline
\end{tabular}

a **, $P<0.0001$.

Table 2. Pearson's correlation coefficients for the four sheath blight evaluation methods used in the assessment of 65 rice genotypes. Data used in these analyses are presented in Supplementary Table S2. ${ }^{\mathrm{a}}$

\begin{tabular}{lcccc}
\hline & $\begin{array}{c}\text { Average } \\
\text { lesion } \\
\text { length }\end{array}$ & $\begin{array}{c}\text { Average } \\
\text { visual } \\
\text { rating }\end{array}$ & $\begin{array}{c}\text { Maximum } \\
\text { lesion } \\
\text { length }\end{array}$ & $\begin{array}{c}\text { Maximum } \\
\text { visual } \\
\text { rating }\end{array}$ \\
\hline Average lesion length & - & - & - & - \\
Average visual rating & $0.80^{* *}$ & - & - & - \\
Maximum lesion length & $0.93^{* *}$ & $0.71^{* *}$ & - & - \\
Maximum visual rating & $0.80^{* *}$ & $0.97^{* *}$ & $0.74^{* *}$ & - \\
\hline
\end{tabular}

a **, $P<0.0001$. and visual rating for 56 genotypes). By analyzing these data, it was clear that disease resistance in specific genotypes had a high level of variation in different environments (Figs. 1 and 2; Supplementary Figures S1 and S2). Correlation analysis revealed significant positive correlations between the average lesion length data of the 169 genotypes in different environments but the correlation coefficients were low, ranging from 0.16 to 0.54 (Table 3 ), with all the correlation coefficients below 0.45 except for two between May 2012 and May 2016, and between May 2015 and May 2016, respectively (Table 3). Only two significantly positive correlations were detected from the average lesion length measurements in 57 genotypes during the 4-year study, but these correlation coefficients were 0.44 and 0.38 , respectively (Table 4). The correlation coefficients were also low when we used the average visual rating data (Supplementary Tables S5 and S6). These low correlation coefficients coincide with the variation in SB resistance level observed in different environments.

Genotype by environment interaction influences SB resistance. Two-way ANOVA of lesion length data from 169 genotypes grown over six environments indicated that the lesion length in different genotypes was significantly different from each other $(\mathrm{F}=19.8, P<$ 0.0001 ), and that lesion length on plants grown in different environments was also significantly different $(\mathrm{F}=1,820.5, P<0.0001)$; the interaction between the genotype and environment was highly significant $(\mathrm{F}=5.0, P<0.0001$; Table 5). The environment contributed $54.6 \%$ to the total variance and the interaction between the genotype and environment contributed to $25.4 \%$ of the total variance, while the genotype only contributed $20 \%$ to the total variance. This explains why disease resistance in the same rice genotype varied so greatly in different environments.

Two-way ANOVA using the visual rating data from 169 genotypes cultivated over six environments suggested that the genotype by environment interaction had a highly significant $(P<0.0001)$

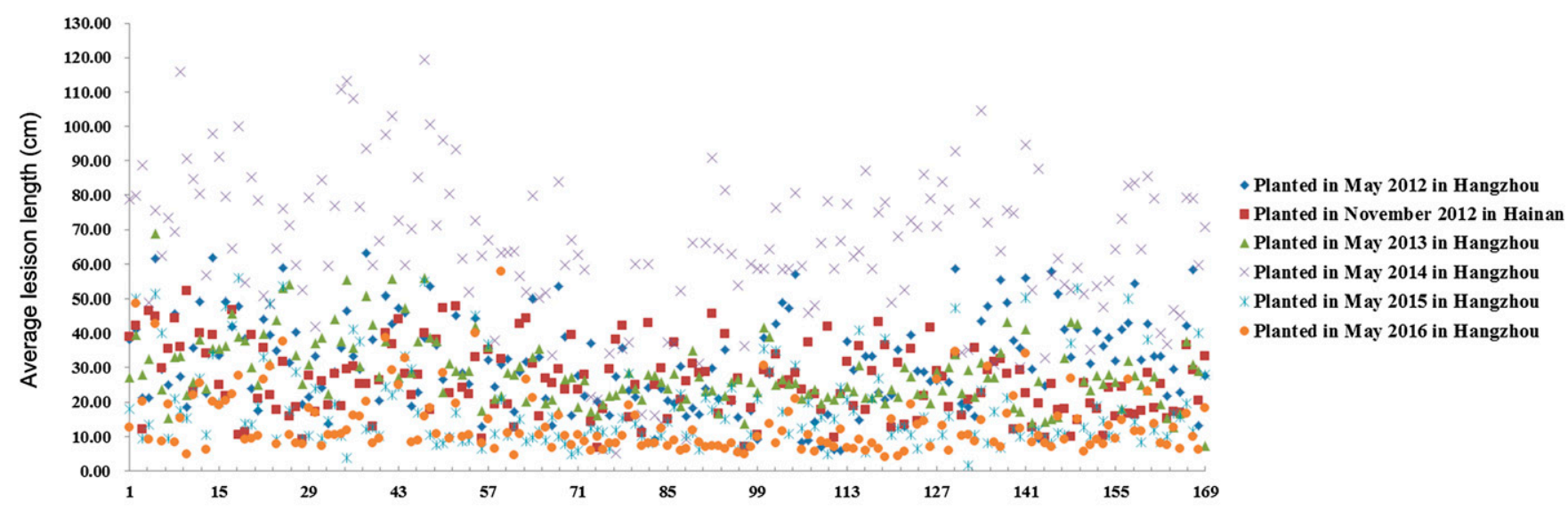

169 rice genotypes evaluated for sheath blight disease resistance in six years/environments

Fig. 1. Scatter diagram showing the average lesion length of 169 rice genotypes evaluated in six environments.

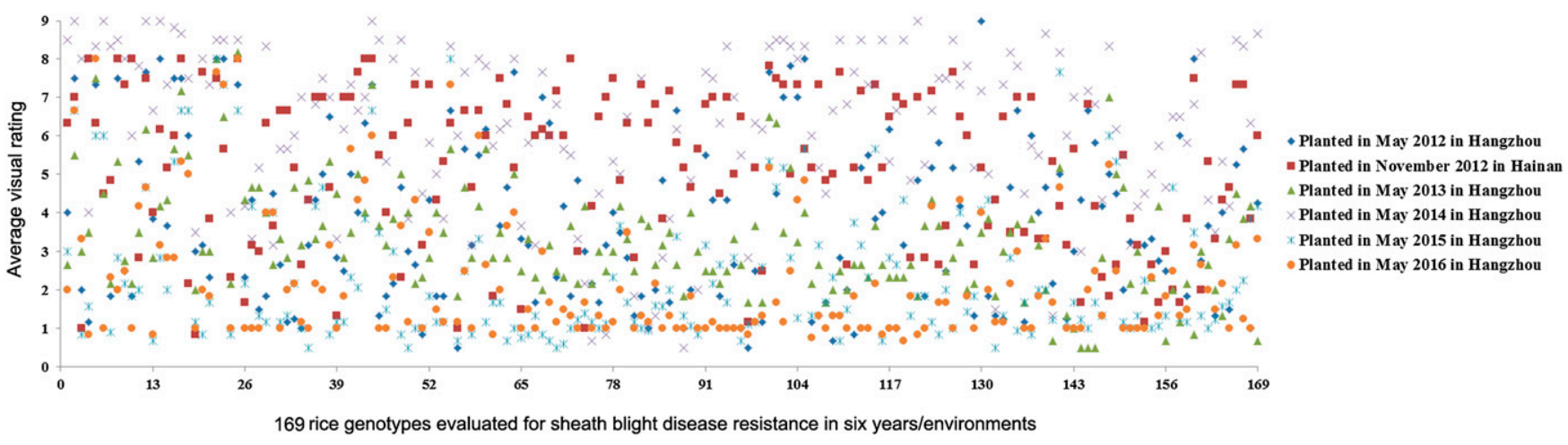

Fig. 2. Scatter diagram showing the average visual rating of 169 rice genotypes evaluated in six environments. 
influence on disease resistance, which contributed $29.8 \%$ of the total variance, while the environment contributed $37.7 \%$ to the total variance and the genotype contributed $32.5 \%$ to the total variance (Table 6).

Two-way ANOVA was also performed on the lesion length data from 57 genotypes cultivated over four environments. This analysis indicated that the genotype by environment interaction had a highly significant influence on disease resistance $(P<0.0001)$ that contributed to $19.8 \%$ of the total variance, while the environment contributed $65.5 \%$ to the total variance and the genotype only contributed to $14.7 \%$ of the total variance (Table 7). Two-way ANOVA of the visual rating data of 56 genotypes over four environments indicated that the genotype by environment interaction had a highly significant influence on disease resistance $(P<0.0001)$ and contributed to $21.9 \%$ of the total variance. The environment contributed $47 \%$ and the genotype contributed to $31.1 \%$ of the total variance, respectively (Table 8).

Of the 169 genotypes analyzed for SB, there were five different environments (different years on the same farm) starting in May in Hangzhou between 2012 and 2016, and one environment in Hainan, November 2013. Two-way ANOVA of the five-environment data from Hangzhou indicated that the genotype by environment interaction had a highly significant effect on SB field resistance, as measured by lesion length or visual ratings (Supplementary Tables S7 and S8). Additionally, the environment contributed more than the genotype to the determination of SB resistance.

From the resistance data of the 56 genotypes cultivated over four environments, we also used the three-environment data from Hangzhou for two-way ANOVA. These data also indicated that the environment had more influence than the genotype on the determination of SB field resistance in rice (Supplementary Tables S9 and S10).
The most stable and unstable genotypes among the 211 genotypes grown over six environments. The standard deviation (SD) and average SD of each of the 211 genotypes in all the six cultivation environments show the stability of SB resistance in the studied rice genotypes. The five most stable genotypes sorted by average lesion SD were Conglaai (2.8), Xueheaizao (3.1), Teai (3.2), Nipponbare (3.6), and IR40 (3.7); and the five most unstable genotypes were Zijindou (17.4), Laolaiqing (17.1), Shenglixian (15.9), Tianjidu (15.6), and Katy (15.1). According to the average SD of visual rating over six environments, the five most stable genotypes were Kongyu131 (0.21), Haobian1 (0.22), Laobaishu (0.23), Teai (0.23), and Tetep (0.29); the five most unstable genotypes were Shenglixian (1.96), Qifozhan (1.77), Vista (1.71), Zhongzao39 (1.67), and B5580A (1.63).

The most stable and unstable genotypes among the 65 genotypes grown over four environments. Based on the SD of each of the 65 genotypes grown in all four environments, and the average SD of the 65 genotypes over four environments, the five most stable genotypes were Xiushui03 (1.7), TianfengB (2.4), Xiushuil03 (2.5), Minghui725 (3.1), and Jin23B (3.1), when sorted by average SD of lesion length. The five most unstable genotypes were Baidao (15.5), L100 (12.7), Gaogudimi (11.6), P1 (11.5), and R-8006 (11.5) according to the sorting of average SD of disease lesions. Finally, the five most stable genotypes were Xiushui103 (0.06), Xiushui03 (0.14), Jin23B (0.17), Hanyangli2 (0.22), and Neixiang85B (0.26), and the five most unstable genotypes were P1 (1.56), R-8006 (1.52), Resormain1 (1.44), Baidao (1.44), and 9308 (1.40) according to the sorting of average SD of visual rating.

Cluster analysis of SB resistance. Six variables were used in cluster analysis. The 211 genotypes were grouped into four clusters:

Table 3. Pearson's correlation coefficients for the average lesion lengths in 169 genotypes grown in six different environments ${ }^{\mathrm{a}}$

\begin{tabular}{|c|c|c|c|c|c|c|}
\hline & $\begin{array}{l}\text { Planted in } \\
\text { May } 2012\end{array}$ & $\begin{array}{c}\text { Planted in } \\
\text { November } 2012\end{array}$ & $\begin{array}{l}\text { Planted in } \\
\text { May } 2013\end{array}$ & $\begin{array}{l}\text { Planted in } \\
\text { May } 2014\end{array}$ & $\begin{array}{l}\text { Planted in } \\
\text { May } 2015\end{array}$ & $\begin{array}{l}\text { Planted in } \\
\text { May } 2016\end{array}$ \\
\hline Planted in May 2012 & - & - & - & - & - & - \\
\hline Planted in November 2012 & $0.22 * *$ & - & - & - & - & - \\
\hline Planted in May 2013 & $0.45^{* *}$ & $0.18^{*}$ & - & - & - & - \\
\hline Planted in May 2014 & $0.40 * *$ & $0.29 * *$ & $0.41 * *$ & - & - & - \\
\hline Planted in May 2015 & $0.35^{* *} *$ & $0.16^{*}$ & $0.45^{* *}$ & $0.29 * *$ & - & - \\
\hline Planted in May 2016 & $0.52 * *$ & $0.25^{* *}$ & $0.43^{* *}$ & $0.33 * *$ & $0.54 * *$ & - \\
\hline
\end{tabular}

a $* *, P<0.01 ; *, P<0.05$.

Table 4. Pearson's correlation coefficients of the average lesion length data of the 57 genotypes grown in four different years ${ }^{\text {a }}$

\begin{tabular}{lccc}
\hline & Planted in November 2013 & Planted in May 2014 & Planted in May 2015 \\
\hline Planted in November 2013 & - & - & - \\
Planted in May 2014 & 0.06 & - & - \\
Planted in May 2015 & 0.08 & $0.44_{*}^{*}$ & - \\
Planted in May 2016 & -0.05 & $0.38^{* *}$ & - \\
\hline
\end{tabular}

a $* *, P<0.01$.

Table 5. Two-way analysis of variance using the lesion length data from 169 genotypes over six different cultivation environments

\begin{tabular}{lrrrrr}
\hline & DF & Type 1 SS & Mean square & F value & $\begin{array}{c}\text { \% of genotype + environment + } \\
\text { genotype } \times \text { environment }\end{array}$ \\
\hline Genotype & 168 & $330,640.7$ & $1,968.1$ & 19.8 & $<0.0001$ \\
Environment & 5 & $904,781.6$ & $180,956.3$ & $1,820.5$ & $<0.0001$ \\
Genotype $\times$ environment & 840 & $420,062.0$ & 500.1 & 5.0 & $<0.0001$ \\
\hline
\end{tabular}

Table 6. Two-way analysis of variance using the visual rating data from 169 genotypes grown in six different environments

\begin{tabular}{lccccc}
\hline & DF & Type 1 SS & Mean square & F value & $\begin{array}{c}\text { \% of genotype + environment + } \\
\text { genotype } \times \text { environment }\end{array}$ \\
\hline Genotype & 168 & $5,300.7$ & 31.6 & 26.7 & $<0.0001$ \\
Environment & 5 & $6,154.2$ & $1,230.8$ & $1,040.6$ & $<0.0001$ \\
Genotype $\times$ environment & 840 & $4,862.3$ & 5.8 & 4.9 & $<0.0001$ \\
\hline
\end{tabular}


group 1 with 20 genotypes, group 2 with 86 genotypes, group 3 with eight genotypes, and group 4 with 97 genotypes (Supplementary Figure S3). Group 3 had the highest SB resistance level, while rice lines in group 1 were the most susceptible to the disease. The groups with
SB resistance from high to low were group $3>$ group $4>$ group $2>$ group 1. Group 3 contained GD66 and Yangdao 4; GD66 was the most resistant genotype identified in this study and Yangdao 4 has been used as an SB-resistant parent in a previous study that mapped

Table 7. Two-way analysis of variance of lesion length in 57 genotypes from four different environments

\begin{tabular}{lrrrrr}
\hline & DF & Type 1 SS & Mean square & F value & $\begin{array}{c}\text { \% of genotype + environment + } \\
\text { genotype } \times \text { environment }\end{array}$ \\
\hline Genotype & 56 & $54,945.4$ & 981.2 & 10.6 & $<0.0001$ \\
Environment & 3 & $244,881.7$ & $81,627.2$ & 878.6 & $<0.0001$ \\
Genotype $\times$ environment & 168 & $73,804.6$ & 439.3 & 4.7 & $<0.0001$ \\
\hline
\end{tabular}

Table 8. Two-way analysis of variance of visual rating data of 56 genotypes from four different environments

\begin{tabular}{lrccccc}
\hline & DF & Type 1 SS & Mean square & F value & P & $\begin{array}{c}\text { \% of genotype + environment + } \\
\text { genotype } \times \text { environment }\end{array}$ \\
\hline Genotype & 55 & $1,450.9$ & 26.4 & 19.8 & $<0.0001$ & 31.1 \\
Environment & 3 & $2,192.1$ & 730.7 & 548.5 & $<0.0001$ & 47.0 \\
Genotype $\times$ environment & 165 & $1,020.9$ & 6.2 & 4.6 & $<0.0001$ & 21.9 \\
\hline
\end{tabular}

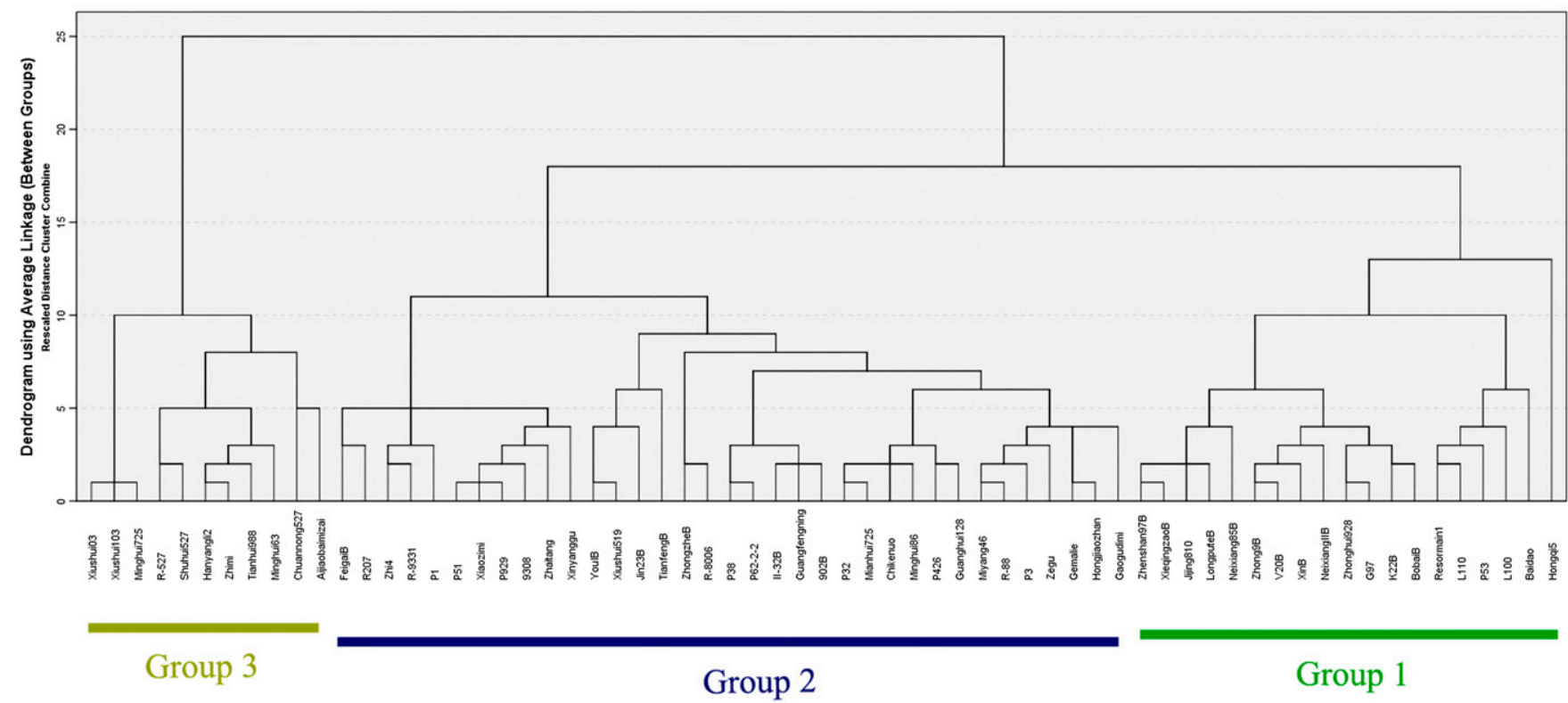

Fig. 3. Cluster analysis of 65 rice genotypes with rescaled distance based on the six variables listed in Supplementary Table S2.

Table 9. The average lesion length, average visual rating, maximum lesion length, and maximum visual rating of the 211 genotypes evaluated in six different environments. The missing data from some genotypes that were omitted and not used in analysis are listed in Supplementary Table S3.

\begin{tabular}{|c|c|c|c|c|c|c|}
\hline 211 genotypes & $\begin{array}{l}\text { Planted in } \\
\text { May } 2012\end{array}$ & $\begin{array}{c}\text { Planted in } \\
\text { November } 2012\end{array}$ & $\begin{array}{l}\text { Planted in } \\
\text { May } 2013\end{array}$ & $\begin{array}{l}\text { Planted in } \\
\text { May } 2014\end{array}$ & $\begin{array}{l}\text { Planted in } \\
\text { May } 2015\end{array}$ & $\begin{array}{l}\text { Planted in } \\
\text { May } 2016\end{array}$ \\
\hline Average lesion length $(\mathrm{cm})$ & 31.0 & 26.4 & 29.1 & 65.1 & 19.4 & 14.3 \\
\hline Average visual rating & 3.7 & 5.2 & 3.4 & 6.4 & 2.4 & 2.3 \\
\hline Maximum lesion length $(\mathrm{cm})$ & 45.5 & 38.1 & 34.5 & 72.4 & 25.8 & 25.5 \\
\hline Maximum visual rating & 4.5 & 6.0 & 3.9 & 6.9 & 3.2 & 3.1 \\
\hline
\end{tabular}

Table 10. The average lesion length, average visual rating, maximum lesion length, and maximum visual rating of the 65 genotypes evaluated in four environments. The missing data of some genotypes that were omitted and not used in analysis are listed in Supplementary Table S4.

\begin{tabular}{lcccc}
\hline 65 genotypes & Planted in November 2013 & Planted in May 2014 & Planted in May 2015 & Planted in May 2016 \\
\hline Average lesion length $(\mathrm{cm})$ & 45.2 & 61.1 & 24.3 & 17.3 \\
Average visual rating & 6.6 & 6.3 & 3.0 & 2.8 \\
Maximum lesion length $(\mathrm{cm})$ & 52.0 & 68.3 & 31.3 & 3.3 \\
Maximum visual rating & 7.3 & 7.0 & 3.8 & 3.6 \\
\hline
\end{tabular}


SB resistance QTLs (Wen et al. 2015). Group 4 contained 9311, Baiyeqiu, and Tetep rice genotypes. The 9311 genome has been sequenced, Baiyeqiu has been used as a SB-resistant parent for mapping resistance QTLs (Xu et al. 2011), and Tetep is an SB-resistant genotype also used in a QTL mapping study (Channamallikarjuna et al. 2010). Group 2 contained TeQing, Jasmine 85, Lemont, and Nipponbare. TeQing and Jasmine 85 have been used in QTL mapping as SB-resistant parents (Pinson et al. 2005; Prasad and Eizenga 2008; Zou et al. 2000), Lemont is generally believed to be a SB susceptible cultivar that is frequently used in QTL mapping, and the International Rice Genome Sequencing Project has sequenced Nipponbare (International Rice Sequencing Project 2005). Group 1 contained Maybelle, which is a cultivar that has been used as a susceptible parent in QTL analysis (Xu et al. 2011).

The cluster analysis using six variables grouped the 65 genotypes into three clusters (Fig. 3). The SB resistance from high to low was as follows: group $3>$ group $2>$ group 1 . The 17 maintainer lines clustered into the susceptible groups 1 and 2; group 1 contained 10 maintainer lines and group 2 contained seven maintainer lines. Six restorer lines were collected in group 3, which included Minghui725, R-527, Shuhui527, Tianhui988, Minghui63, and Chuannong527.

Average resistance levels of the 211 and 65 genotypes in different years. By analyzing the average resistance levels of the 211 genotypes from different years, we found that genotypes had the most resistant level when planted in May 2016, and were the most susceptible to disease when planted in May 2014 (Table 9). The resistance levels of the 65 genotypes during different years are listed in Table 10

\section{Discussion}

Rice SB is the most difficult disease to study among the three major rice diseases due to the complex nature of SB resistance. No more than 10 QTLs have been confirmed with observable contributions to SB resistance despite extensive efforts in mining for QTLs (Xue et al. 2016). We provide direct evidence in this study that the environment has more of an influence than the genotype on SB field resistance, which would definitely affect QTL mapping results. The results presented in this study explain why the QTL mapping studies of SB field resistance are so difficult to perform and why so little achievement has been attained with map-based cloning strategies despite extensive efforts made in the rice SB resistance communities. It is no surprise that if a QTL was identified in one environment and not in another using the same mapping population, that it is because the environment influences disease resistance more than it does the genotype. Therefore, caution must be taken in the use of QTL fine mapping of SB resistance in the field. Here, we use an example based on our QTL mapping experiences to thoroughly explain this phenomenon. An SB resistance QTL was detected at a specific chromosome location using a $\mathrm{BC}_{4} \mathrm{~F}_{2}$ mapping population with 150 individuals; although one may perform fine-mapping studies using $\mathrm{BC}_{4} \mathrm{~F}_{3}$ descendants with 1,500 or more individuals, it is very likely that the QTL would not be redetected in 1,500 individuals. For the $\mathrm{BC}_{4} \mathrm{~F}_{3}$ mapping populations, we recommend using at least 20 individuals with overlapping chromosome segments spanning the QTL region for fine mapping. These individuals could be used in the evaluation of SB field resistance over three or more continuous years from $\mathrm{BC}_{4} \mathrm{~F}_{3}$ to $\mathrm{BC}_{4} \mathrm{~F}_{5}$ generations if the field rating method is used. Once the QTL region has been confirmed and delimited to a much smaller segment by the multiyear results, segregating individuals with overlapping chromosome segments spanning the smaller QTL region can be reused with the method mentioned above, until the QTL is fine mapped to a suitable chromosome segment for cloning. This method is very similar to that used by Zuo et al. $(2013,2014 a)$, although they did not describe why this strategy was used for their analysis. Another option may be to use $\mathrm{BC}_{4} \mathrm{~F}_{3}$ mapping populations with 1,500 or more segregating individuals with an inoculation and evaluation method that utilizes microchamber screening (Jia et al. 2007), an inoculated detached-leaf method in Petri plates (Molla et al. 2013), or other methods with a more uniform and reproducible response to the SB pathogen (Park et al. 2008).
SB ratings in the field are both labor intensive and time consuming and are confounded by environmental conditions. To obtain accurate field resistance results, we repeated our evaluation of different rice genotypes over multiple years. By evaluating the field resistance of 211 genotypes over six environments and 65 genotypes over four environments, we identified the indica restorer line GD66 as an excellent SB resistance germplasm that would be helpful in the breeding of SB-resistant rice lines. However, the cluster analysis grouped TeQing, Jasmine 85, and Lemont into the same group; TeQing and Jasmine 85 are generally considered to be moderately resistant cultivars that have been used frequently for QTL analysis as resistant parents ( $\mathrm{Li}$ et al. 1995; Pinson et al. 2005; Zou et al. 2000), while Lemont is a well-known susceptible cultivar. It is not surprising that these three cultivars were clustered together based on our data. The fact that the two resistant cultivars TeQing and Jasmine 85 clustered with the susceptible cultivar Lemont may be due to different SB isolates and environments employed by previous researchers.

The results in this study demonstrate that the environment contributes more than the genotype in the determination of SB field resistance. A number of environmental factors such as sunlight, humidity, temperature, soil fertility, and silicon levels in the soil may contribute to the variation of SB field resistance (Park et al. 2008); however, it is still poorly understood which environmental factors contributed the most, which should be the focus of studies in the future.

The present work illustrated that the environment had more effect than the genotype on the determination of SB field resistance and indicated that the interaction between genotype and environment is the greatest obstacle for the precise determination of rice SB field resistance. The results provide crucial information for QTL mapping using SB field resistance data and explain why rice SB disease is so difficult to study. GD66, the most resistant genotype identified in the present work, would be a good candidate for genetic studies and breeding.

\section{Acknowledgments}

This work was supported by the National Key R\&D program (2016YFD0102100), the Zhejiang Provincial Natural Science Foundation (LY16C060002), and a fund from the Science Technology Department of Zhejiang Province to the Zhejiang Agricultural Key Breeding Project (2016C02050-4). We thank Ling Wang and Shiwen Huang at CNRRI for kindly providing the Rhizoctonia solani isolate, ZJ03. We thank Xinghua Wei at National Mid-term Rice Genebank at CNRRI, and Guanfu Fu at CNRRI for kindly providing most of the rice genotypes used in this study. We thank Editage (www.editage.cn) for English language editing.

\section{Literature Cited}

Bonman, J. M. 1992. Durable resistance to rice blast disease - environmental influences. Euphytica 63:115-123.

Channamallikarjuna, V., Sonah, H., Prasad, M., Rao, G. J. N., Chand, S., Upreti, C., Singh, N. K., and Sharma, T. R. 2010. Identification of major quantitative trait loci $q S B R 11-1$ for sheath blight resistance in rice. Mol. Breed. 25:155-166.

Egesi, C. N., Onyeka, T. J., and Asiedu, R. 2009. Environmental stability of resistance to anthracnose and virus diseases of water yam (Dioscorea alata). Afr. J. Agric. Res. 4:113-118.

Ghosh, P., Sen, S., Chakraborty, J., and Das, S. 2016. Monitoring the efficacy of mutated Allium sativum leaf lectin in transgenic rice against Rhizoctonia solani. BMC Biotechnol. 16:24.

Groth, D. E., and Bond, J. A. 2007. Effects of cultivars and fungicides on rice sheath blight, yield, and quality. Plant Dis. 91:1647-1650.

International Rice Sequencing Project. 2005. The map-based sequence of the rice genome. Nature 436:793-800.

Jia, Y., Correa-Victoria, F., McClung, A., Zhu, L., Liu, G., Wamishe, Y., Xie, J., Marchetti, M. A., Pinson, S. R. M., Rutger, J. N., and Correll, J. C. 2007. Rapid determination of rice cultivar responses to the sheath blight pathogen Rhizoctonia solani using a micro-chamber screening method. Plant Dis. 91: 485-489.

Kilic, H., Sagir, A., and Bayram, Y. 2009. Estimates of genotype $\times$ environment interactions and heritability of black point in durum wheat. Not. Sci. Biol. 1:92-96.

Li, Z. K., Pinson, S. R. M., Marchetti, M. A., Stansel, J. W., and Park, W. D. 1995. Characterization of quantitative trait loci (QTLs) in cultivated rice contributing to field resistance to sheath blight (Rhizoctonia solani). Theor. Appl. Genet. 91: 382-388.

Lussewa, R. K., Edema, R., and Lamo, J. 2016. Magnitude of genotype $\times$ environment interaction for bacterial leaf blight resistance in rice growing areas of Uganda. Afr. Crop Sci. J. 24:11-24.

Molla, K. A., Karmakar, S., Chanda, P. K., Ghosh, S., Sarkar, S. N., Datta, S. K. and Datta, K. 2013. Rice oxalate oxidase gene driven by green tissue-specific 
promoter increases tolerance to sheath blight pathogen (Rhizoctonia solani) in transgenic rice. Mol. Plant Pathol. 14:910-922.

Park, D. S., Sayler, R. J., Hong, Y. G., Nam, M. H., and Yang, Y. N. 2008. A method for inoculation and evaluation of rice sheath blight disease. Plant Dis. 92:25-29.

Pinnschmidt, H. O., and Hovmøller, M. S. 2002. Genotype xenvironment interactions in the expression of net blotch resistance in spring and winter barley varieties. Euphytica 125:227-243.

Pinson, S. R. M., Capdevielle, F. M., and Oard, J. H. 2005. Confirmation QTLs and finding additional loci conditioning sheath blight resistance in rice using recombinant inbred lines. Crop Sci. 45:503-510.

Prasad, B., and Eizenga, G. C. 2008. Rice sheath blight disease resistance identified in Oryza spp. accessions. Plant Dis. 92:1503-1509.

Richa, K., Tiwari, I. M., Kumari, M., Devanna, B. N., Sonah, H., Kumari, A., Nagar, R., Sharma, V., Botella, J. R., and Sharma, T. R. 2016. Functional characterization of novel chitinase genes present in the sheath blight resistance QTL: qSBR11-1 in rice Line Tetep. Front. Plant Sci. 7:244.

Sibiya, J., Tongoona, P., Derera, J., and Rij, N. V. 2012. Genetic analysis and genotype $\times$ environment $(\mathrm{G} \times \mathrm{E})$ for grey leaf spot disease resistance in elite African maize (Zea mays L.) germplasm. Euphytica 185:349-362.

Srinivasachary, Willocquet, L., and Savary, S. 2011. Resistance to rice sheath blight (Rhizoctonia solani) [teleomorph: Thanatephorus cucumeris (A.B. Frank) Donk.] disease: current status and perspectives. Euphytica 178:1-22.

Talukder, Z. I., McDonald, A. J. S., and Price, A. H. 2005. Loci controlling partial resistance to rice blast do not show marked QTL $\times$ environment interaction when plant nitrogen status alters disease severity. New Phytol. 168:455-464.

Wen, Z. H., Zeng, Y. X., Ji, Z. J., and Yang, C. D. 2015. Mapping quantitative trait loci for sheath blight disease resistance in Yangdao 4 rice. Genet. Mol. Res. 14: 1636-1649.

Xu, Q., Yuan, X. P., Yu, H. Y., Wang, Y. P., Tang, S. X., and Wei, X. H. 2011. Mapping quantitative trait loci for sheath blight resistance in rice using double haploid population. Plant Breed. 130:404-406.
Xue, X., Cao, Z. X., Zhang, X. T., Wang, Y., Zhang, Y. F., Chen, Z. X., Pan, X. B. and Zuo, S. M. 2016. Overexpression of OsOSM1 enhances resistance to rice sheath blight. Plant Dis. 100:1634-1642.

Zeng, Y. X., Ji, Z. J., Ma, L. Y., Li, X. M., and Yang, C. D. 2011. Advances in mapping loci conferring resistance to rice sheath blight and mining Rhizoctonia solani resistant resources. Rice Sci. 18:56-66.

Zeng, Y. X., Ji, Z. J., and Yang, C. D. 2015a. The way to a more precise sheath blight resistance QTL in rice. Euphytica 203:33-45.

Zeng, Y. X., Xia, L. Z., Wen, Z. H., Ji, Z. J., Zeng, D. L., Qian, Q., and Yang, C. D. 2015b. Mapping resistant QTLs for rice sheath blight disease with a doubled haploid population. J. Integr. Agric. 14:801-810.

Zhao, F., and $\mathrm{Xu}, \mathrm{S}$. 2012. Genotype by environment interaction of quantitative traits: a case study in barley. G3-Genes Genom. Genet. 2:779-788.

Zou, J. H., Pan, X. B., Chen, Z. X., Xu, J. Y., Lu, J. F., Zhai, W. X., and Zhu, L. H. 2000. Mapping quantitative trait loci controlling sheath blight resistance in two rice cultivars (Oryza sativa L.). Theor. Appl. Genet. 101:569-573.

Zuo, S. M., Yin, Y. J., Pan, C. H., Chen, Z. X., Zhang, Y. F., Gu, S. L., Zhu, L. H. and Pan, X. B. 2013. Fine mapping of $q S B-11^{L E}$, the QTL that confers partial resistance to rice sheath blight. Theor. Appl. Genet. 126:1257-1272.

Zuo, S. M., Zhang, L., Wang, H., Yin, Y. J., Zhang, Y. F., Chen, Z. X., Ma, Y. Y., and Pan, X. B. 2008. Prospect of the QTL-qSB-9 ${ }^{\mathrm{Tq}}$ utilized in molecular breeding program of japonica rice against sheath blight. J. Genet. Genomics 35:499-505.

Zuo, S. M., Zhang, Y. F., Yin, Y. J., Li, G. Z., Zhang, G. W., Wang, H., Chen, Z. X., and Pan, X. B. 2014a. Fine-mapping of $q S B-9^{T Q}$, a gene conferring major quantitative resistance to rice sheath blight. Mol. Breed. 34:21912203.

Zuo, S. M., Zhu, Y. J., Yin, Y. J., Wang, H., Zhang, Y. F., Chen, Z. X., Gu, S. L., and Pan, X. B. 2014b. Comparison and confirmation of quantitative trait loci conferring partial resistance to rice sheath blight on chromosome 9. Plant Dis. 98:957-964. 